\title{
Deletion of self-reactive CCR7- thymocytes in the absence of MHC expression on thymic epithelial cells
}

\author{
Rushika C. Wirasinha ${ }^{1} \cdot$ Anna Chan ${ }^{1} \cdot$ Jin Yan Yap ${ }^{2}$. Daniel Y. Hu Charis E. Teh $^{3,4} \cdot$ Daniel H. D. Gray ${ }^{3,4}$. \\ Christopher C. Goodnow ${ }^{5,6}$. Stephen R. Daley ${ }^{1}$
}

Received: 17 September 2018 / Revised: 18 March 2019 / Accepted: 5 April 2019 / Published online: 24 April 2019

(c) ADMC Associazione Differenziamento e Morte Cellulare 2019

\begin{abstract}
The selection of $\alpha \beta \mathrm{T}$ cells in the thymus is punctuated by checkpoints at which thymocytes differentiate or undergo apoptosis. Wave 1 deletion is defined as apoptosis within nascent $\alpha \beta$ T-cell antigen receptor (TCR)-signalled thymocytes that lack CCR7 expression. The antigen-presenting cell (APC) types that mediate wave 1 deletion are unclear. To measure wave 1 deletion, we compared the frequencies of TCR $\beta+$ CD $5+$ Helios + CCR7- cells in nascent thymocyte cohorts in mice with normal or defective apoptosis. This thymocyte population is small in mice lacking major histocompatibility complex (MHC) expression. The scale of wave 1 deletion was increased by transgenic expression of the self-reactive Yae62 TCR $\beta$ chain, was almost halved when haemopoietic APCs lacked MHC expression and, surprisingly, was unchanged when epithelial cells lacked MHC expression. These findings demonstrate efficiency, and some redundancy, in the APC types that mediate wave 1 deletion in the normal mouse thymus.
\end{abstract}

Edited by D.R. Green

Supplementary information The online version of this article (https:// doi.org/10.1038/s41418-019-0331-8) contains supplementary material, which is available to authorized users.

Stephen R. Daley

stephen.daley@monash.edu

1 Infection and Immunity Program, Monash Biomedicine Discovery Institute and Department of Biochemistry and Molecular Biology, Monash University, Melbourne, VIC, Australia

2 Department of Immunology and Infectious Disease, The John Curtin School of Medical Research, The Australian National University, Canberra, ACT, Australia

3 Walter and Eliza Hall Institute of Medical Research, Melbourne, VIC, Australia

4 Department of Medical Biology, University of Melbourne, Melbourne, VIC, Australia

5 Immunology Division, Garvan Institute of Medical Research, Sydney, NSW, Australia

6 St Vincent's Clinical School, University of New South Wales, Sydney, NSW, Australia

\section{Introduction}

Unfit thymocytes undergo apoptosis at four checkpoints in thymic $\alpha \beta$ T-cell development. After thymocytes that fail to assemble a functional TCR $\beta$ chain are removed, those that fail to express a TCR that engages a self-antigen/MHC ligand die by neglect at the next checkpoint. Of the thymocytes that pass the death-by-neglect checkpoint, most undergo apoptosis before or after upregulating the chemokine receptor, CCR7, at the checkpoints called wave 1 and wave 2 of deletion, respectively [1-3]. Thymocytes are thought to require TCR engagement with self-antigen/MHC on cortical thymic epithelial cells (cTEC) to pass the deathby-neglect checkpoint. This is based on the reduction in CD4 + CD8 - single positive (SP) and CD8SP thymocytes when cTEC, or all non-haemopoietic cells, lack MHC expression $[4,5]$. However, as death by neglect and wave 1 deletion both occur in CCR7- thymocytes at the CD4+ CD8 + double positive (DP) stage [6,7], analysis of CD4 and CD8 expression is not sufficient to distinguish cells destined for these fates.

Inhibition of the intrinsic apoptosis pathway delays death by neglect, but is not sufficient to allow differentiation in thymocytes expressing a TCR that cannot engage a selfantigen/MHC ligand [8]. Inhibition of apoptosis also rescues thymocytes from deletion, revealing that $55-57 \%$ of 
TCR-signalled thymocytes are deleted within the DP stage [7] or at wave 1 [2]. Another study measured the dynamics of thymocyte progression or death throughout development and found that $67 \%$ of TCR-signalled thymocytes are deleted within the DP stage [6]. This study also analysed thymic selection in chimeric mice lacking MHC class I (MHCI) or MHCII expression in non-haemopoietic cells [6]. However, as all chimeras expressed MHC in haemopoietic cells, the possibility that haemopoietic APC had induced TCR signalling in a portion of thymocytes was not excluded.

High expression of the transcription factor, Helios, marks $\alpha \beta$ TCR + thymocytes that are susceptible to deletion [2]. We previously determined the timing of the two waves of deletion in thymocyte development by labelling proliferating precursor cells with 5-ethynyl-2'-deoxyuridine (EdU) and tracking the frequency of EdU + cells in the Helios + CCR7- and Helios + CCR7 + thymocyte populations over time [3]. Here, we focused on EdU + thymocytes and measured the percentage of these cells stimulated to upregulate Helios or CCR7 in mice with normal or defective apoptosis. We found that expression of the Yae62 TCR $\beta$ chain transgene [9] increased the scale of wave 1 deletion, indicating that the normal thymic APC network is not a rate-limiting component of wave 1 deletion. The scale of wave 1 deletion was normal when non-haemopoietic cells lacked MHC expression, was almost halved when haemopoietic cells lacked MHC expression and was negligible when both APC types lacked MHC expression.

\section{Results}

\section{Definition of cells undergoing wave 1 deletion}

The majority of proliferating cells in the thymus are in the outer cortex at the stage immediately preceding $\alpha \beta$ TCR expression [10-12]. To label nascent thymocytes, we injected C57BL/6 (B6) mice, as well as apoptosis-deficient mice carrying a transgene encoding human BCL2 (BCL2$\operatorname{tg}$ ) [13], with a single dose of EdU 1-3 days before thymocytes were analysed by flow cytometry. This time course was selected because the peak of wave 1 deletion occurs $\sim 2$ days after proliferation [3]. The frequency of nascent thymocytes, which were defined as EdU + cells expressing CD24 (Fig. 1a), a marker of thymocyte immaturity [12], was not significantly different between B6 and BCL2-tg groups (Fig. 1d). As thymocytes upregulate TCR $\beta$ and CD5 within 1 day after the onset of TCR signalling, TCR $\beta$ and CD5 have been used to subdivide DP thymocytes into TCRsignalled and non-TCR-signalled subsets [6, 14]. The frequency of TCR $\beta+\mathrm{CD} 5+$ cells in the $\mathrm{EdU}+\mathrm{CD} 24+$ population increased from 1 to 3 days after EdU injection similarly in B6 and BCL2-tg mice (Fig. 1b, e). While the nascent TCR-signalled population was comprised mainly of Helios-CCR7- cells throughout this short time course, the Helios- CCR7 + cell frequency increased similarly in B6 and BCL2-tg groups (Fig. 1c, f). However, BCL2-tg expression resulted in a significant increase in the Helios + CCR7- cell frequency (Fig. 1c, g). At 2-3 days after EdU injection, the difference in Helios + CCR7- cell frequency was approximately tenfold, suggesting that $\sim 90 \%$ of the CCR7- thymocytes that were stimulated to upregulate Helios in B6 mice had undergone wave 1 deletion.

BCL2-tg expression in $\mathrm{B}^{-\mathrm{m}^{-/}} \mathrm{H} 2-\mathrm{Aa}^{-/-}$mice enables the formation of a substantial Helios-CCR7 + thymocyte population [3], questioning the use of Helios and CCR7 as markers to distinguish TCR-signalled cells from non-TCRsignalled cells. Here, the use of EdU and CD24 to identify nascent thymocytes, combined with TCR $\beta$ and CD5 as markers of TCR signalling [6, 14] improved the specificity of detection of thymocytes that had received a productive TCR signal, as few EdU + CD24 + TCR $\beta+$ CD5 + cells were detected in BCL2-tg $B 2 \mathrm{~m}^{-/-} \mathrm{H} 2-A a^{-/-}$mice (Fig. 1b).

While most thymocytes that receive a strong TCR signal at the DP stage are deleted, some cells survive this type of stimulation. These thymocytes downregulate CD4 and CD8, upregulate PD-1 and begin to differentiate into intestinal CD $8 \alpha \alpha$ intraepithelial lymphocytes (IEL) [1518]. Many newly formed Helios + CCR7- thymocytes in BCL2-tg mice downregulate CD4 and CD8 and upregulate PD-1 [3]. This applies also to B6 mice, as most EdU + Helios + CCR7- thymocytes were CD4-CD8- double negative (DN) cells (Fig. 1h) expressing more PD-1 than EdU + Helios- CCR7 + thymocytes (Fig. 1i). Most CCR7thymocytes that survive the process of Helios upregulation thus attain a DN PD-1 + phenotype.

\section{Wave 1 deletion phenotypes in both Type $A$ and Type B IELp}

The DN thymocyte population contains two types of IEL precursors (IELp) $[19,20]$. Type A IELp are PD-1 + and reside in the thymic cortex whereas Type B IELp are NK1.1 + and are in the thymic medulla [20]. Compared with B6 mice, BCL2-tg mice had increased numbers of Type A IELp, while Type B IELp numbers also tended to be higher without reaching statistical significance (Fig. 2a). Most Type A IELp were Helios + in both mouse strains, but BCL2-tg expression markedly increased the frequency of Helios + cells among Type B IELp (Fig. 2b). CD24 was expressed on Type A IELp but not on Type B IELp (Fig. 2b), consistent with Type A IELp being developmentally younger [20]. Few cells in either subset expressed CCR7, regardless of BCL2-tg expression (Fig. 2c). These findings suggest that BCL2-tg expression 


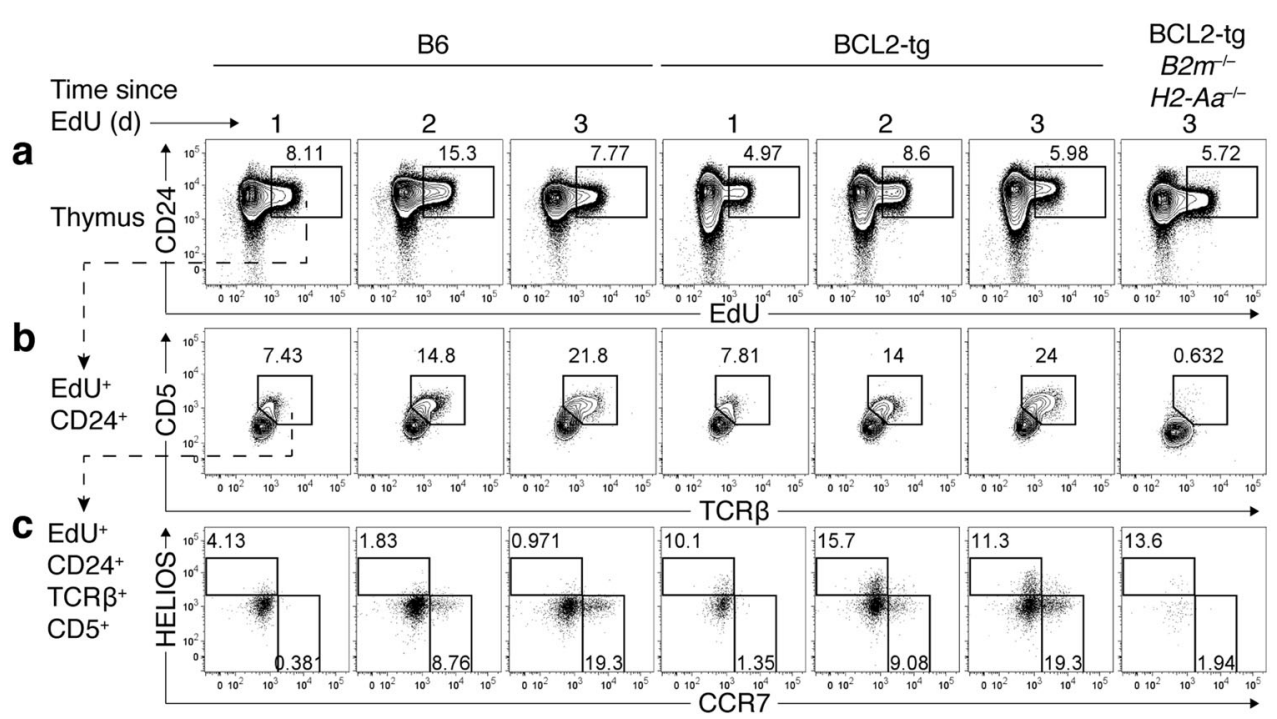

d

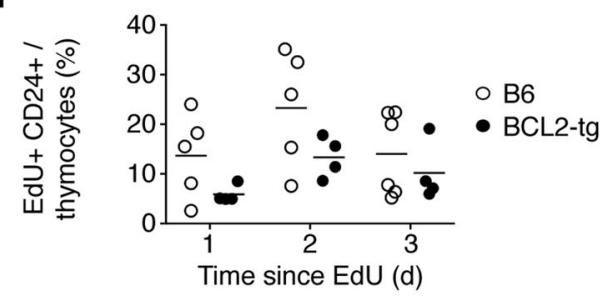

f

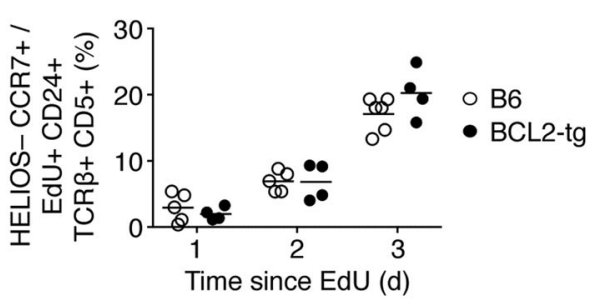

h

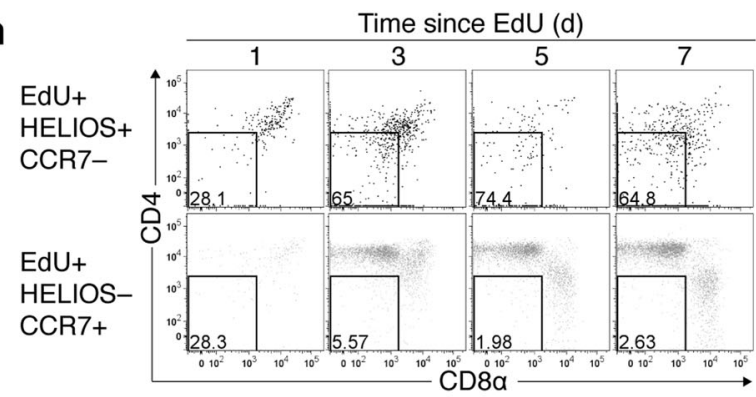

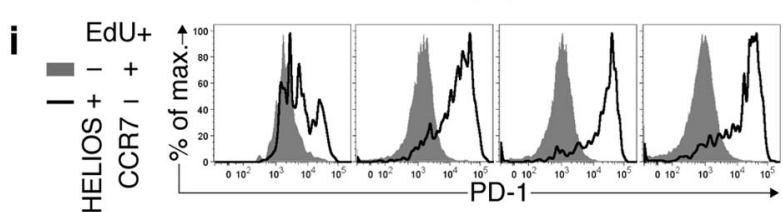

e
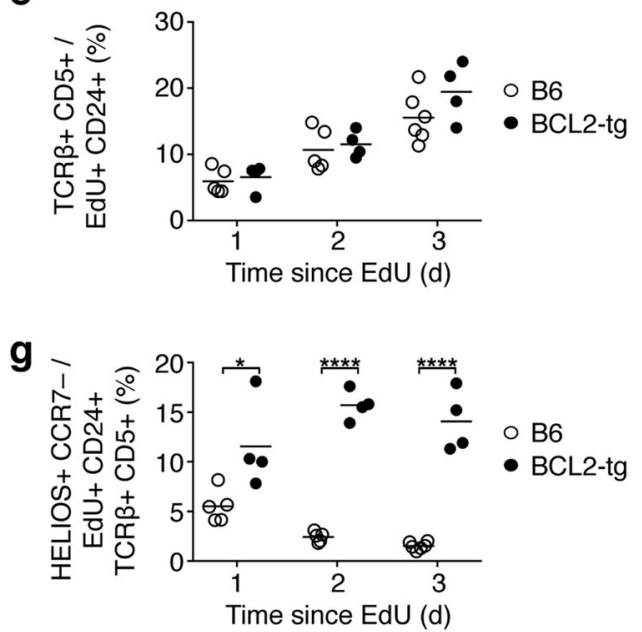

O EdU+ HELIOS+ CCR7-
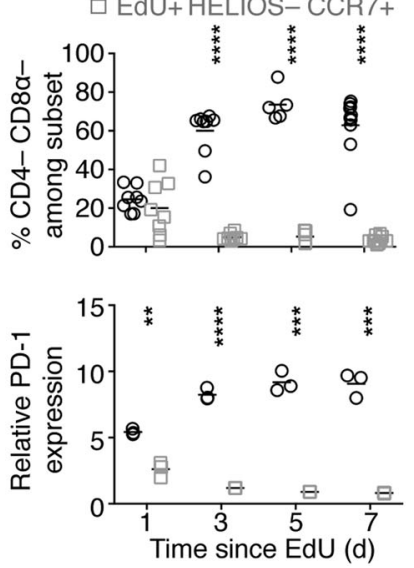
Fig. 1 Definition of nascent thymocytes stimulated to undergo wave 1 deletion. B6, BCL2-tg or BCL2-tg B2m ${ }^{-/-} H 2-A a^{-l-}$ mice (denoted at top) received a single EdU injection i.v. 1, 2 or 3 days before thymocytes were analysed by flow cytometry. Representative plots show a CD24 vs EdU on thymocytes, $\mathbf{b}$ CD5 vs TCR $\beta$ on EdU + CD $24+$ thymocytes, and $\mathbf{c}$ Helios vs CCR7 on EdU + CD24 + TCR $\beta+$ CD5 + thymocytes. Numbers on plots indicate the percentage of events within the gates. Graphs show the percentage of $\mathbf{d}$ EdU $+\mathrm{CD} 24+$ events as in (a); e TCR $\beta+$ CD5 + events as in (b); f Helios - CCR7 + or $\mathbf{g}$ Helios + CCR7- events as in (c). h From B6 mice injected once with EdU 1, 3, 5 or 7 days before analysis, plots show CD4 vs CD8 $\alpha$ on EdU + Helios + CCR7- or EdU + Helios - CCR7 + thymocytes (left) with a summary showing the percentage of CD4-CD8 $\alpha$ - events (right). i Histograms show PD-1 expression on the subsets shown in (h) with a summary of PD-1 expression normalised to DP thymocytes in the same sample (right). Each symbol represents an individual mouse and lines show the group mean combined from two independent experiments $(\mathbf{d}-\mathbf{h})$ or from one experiment representative of 3 (i). For each time point, a Student's $t$ test was used to compare B6 and BCL2-tg groups with Holm-Sidak's post-test without assuming a consistent standard deviation. $P$ values: $*<0.05 ; \quad * *<0.01$; $* * *<0.001 ; * * * *<0.0001$

rescues would-be Type A IELp and Type B IELp from wave 1 deletion.

\section{Conservation of the TCR self-reactivity hierarchy when apoptosis is inhibited}

As BCL2-tg expression rescues thymocytes from wave 1 deletion, allowing these cells to attain IELp phenotypes, we reasoned that a comparison of B6 and BCL2-tg mice may reveal TCR repertoire features that promote deletion versus IELp differentiation. To our published TCR-sequence database comprising nine T-cell subsets from the thymus, spleen or small intestine of B6 mice [21] we added Type B IELp data from B6 mice, as well as all ten subsets from BCL2-tg mice (Supplementary Figure 1). First, we examined usage of a set of hydrophobic, amino acid doublets at positions 6 and 7 (P6-P7) of the TCR complementaritydetermining region 3 (CDR3) [22]. The frequency of CDR3 sequences carrying these P6-P7 doublets is a population-level index of TCR self-reactivity and is called the hydrophobic index hereafter. Independent of BCL2-tg expression, in both TCR $\alpha$ and TCR $\beta$ repertoires, Type A IELp and IEL had higher hydrophobic indices than thymic and splenic CD4 + Foxp3 + T-regulatory cells (T-reg), CD4 + Foxp3- conventional T cells (T-conv) and CD8 + T-conv (Fig. 2d and Supplementary Figure 2). BCL2-tg expression increased the hydrophobic index in the CD4+ T-conv TCR $\beta$ repertoire, as observed in apoptosis-defective $\mathrm{Bim}^{-/}$mice (Fig. 2d) [22]. In BCL2-tg mice, the hydrophobic index was markedly increased in the Type B IELp $\operatorname{TCR} \alpha$ and TCR $\beta$ repertoires and slightly increased in the Type A IELp TCR $\alpha$ repertoire (Fig. 2d). These data provide a new tranche of evidence that many self-reactive thymocytes undergo either wave 1 deletion or IELp differentiation and suggest that wave 1 deletion preferentially eliminates would-be Type B IELp that express strongly selfreactive TCRs.

Next, we enumerated the frequency of CDR3 sequences with cysteine within two positions of the CDR3 apex, called the cysteine index in this paper. In B6 mice, compared with pre-selection thymocytes, the cysteine index is increased in Type A IELp and IEL whereas it is decreased in T-reg, CD4 + T-conv and CD8 + T-conv (Fig. 2e) [21]. In BCL2-tg mice, we found the cysteine index was increased in Type A IELp, IEL and CD4 + T-conv, while Type B IELp showed a similar trend without reaching statistical significance (Fig. 2e). Despite these effects, there were significant correlations between B6 and BCL2-tg mice for both TCR selfreactivity indices (Fig. 2d, e), indicating that the TCR self-reactivity hierarchy is largely conserved when apoptosis is inhibited.

\section{Altered scales of wave 1 deletion in TCR $\beta$ transgenic mice}

Compared with B6 thymocytes, induction of TCR signalling by self-antigen/MHC ligands is more common in thymocytes expressing the Yae62 TCR $\beta$ transgene (Yae62 $\beta$-tg) and less common in thymocytes expressing the B3K506 TCR $\beta$ transgene (B3K506 $\beta-\operatorname{tg}$ ) [22]. To validate our flow-cytometric assay, we analysed B6, Yae62 $\beta$-tg and B3K506 - tg mice, including BCL2-tg versions of each strain. Three days after EdU injection, we measured the induction of TCR-signalled cell subsets as a frequency of the EdU $+\mathrm{CD} 24+$ population, which was not significantly affected by TCR $\beta$ transgene expression (Fig. 3a, b). As expected, Yae62 $\beta$-tg expression increased the TCR $\beta+\mathrm{CD} 5+$ cell frequency (Fig. 3c, d). The mean Helios-CCR7 + cell frequency was $2-4 \%$ in B6 and B3K506 $\beta$-tg groups versus $9 \%$ in Yae62 $\beta$-tg mice, with no change due to BCL2-tg expression (Fig. 3e, g). Yae62 $\beta$-tg expression thus increases the frequency of nascent thymocytes that pass the wave 1 checkpoint without upregulating Helios. While the Helios + CCR7- cell frequency was similar in the B6 and B3K506 $\beta$-tg groups with normal apoptosis, analysis of BCL2-tg mice revealed that B3K506 $\beta$-tg expression causes a significant decrease in wave 1 deletion (Fig. 3e, f). However, BCL2-tg expression was not required to detect the large increase in wave 1 deletion caused by Yae62 $\beta$-tg expression (Fig. 3e, f). These effects of TCR $\beta$ transgene expression demonstrate that the "architecture" of the pre-selection TCR repertoire can modulate cellular flow through the wave 1 checkpoint. 

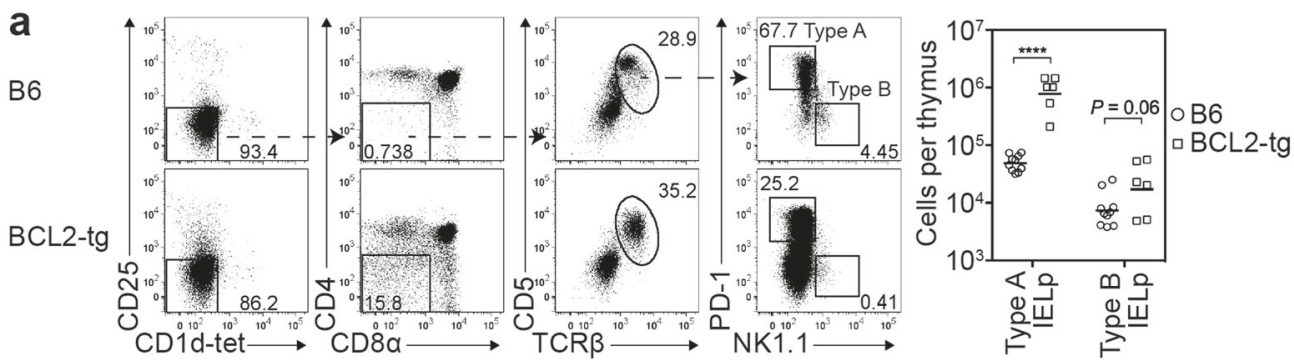

(PBS-44)
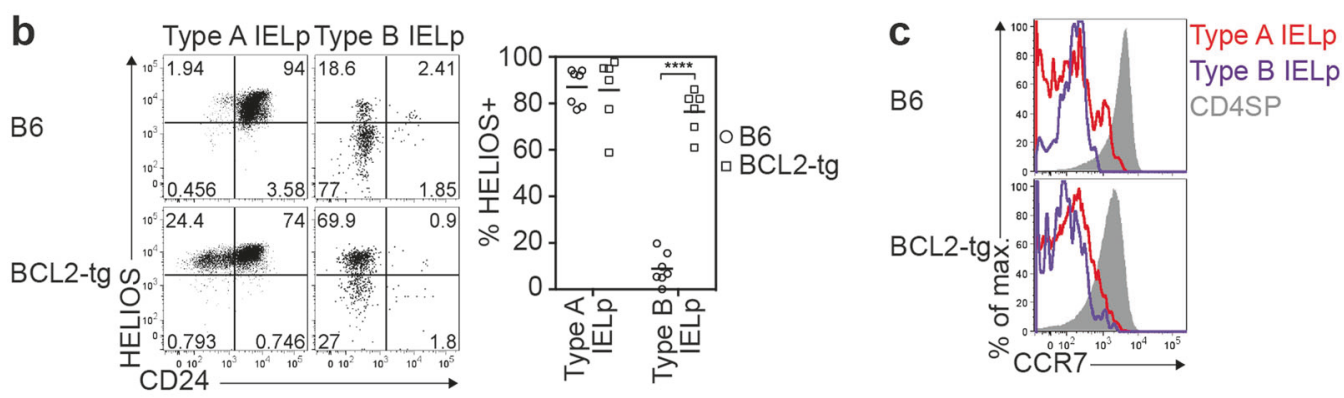

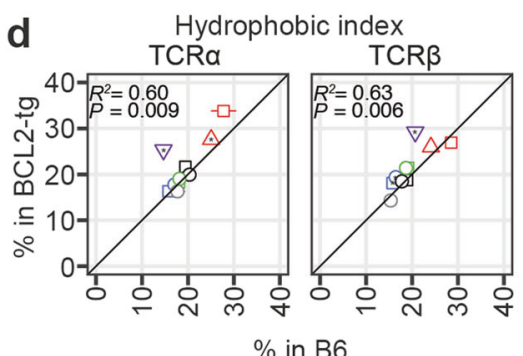

$\%$ in $\mathrm{B} 6$

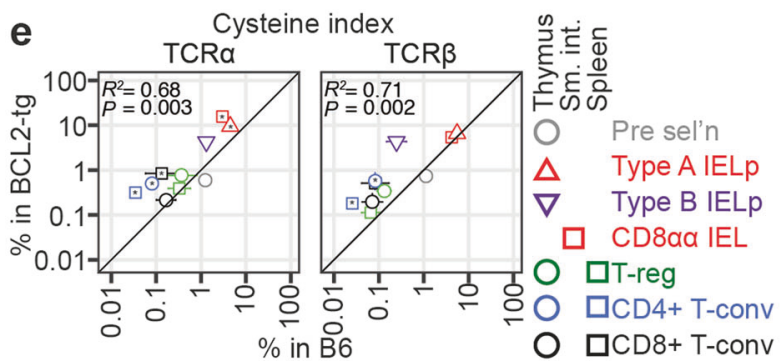

Fig. 2 Features of wave 1 deletion in both Type A and Type B IELp. a After excluding thymocytes bound by anti-CD25 or PBS-44/CD1d tetramer, the CD4-CD8 $\alpha-\mathrm{TCR} \beta+\mathrm{CD} 5+$ population was analysed for expression of PD-1 (to identify Type A IELp) and NK1.1 (to identify Type B IELp), with a summary (right) showing the number of cells in each subset per thymus in B6 and BCL2-tg mice. b Helios/ CD24 phenotypes of Type A and Type B IELp populations gated in (a), with a summary (right) of the percentage of Helios + cells in each subset from multiple mice. Each symbol represents an individual mouse and lines show the group mean compiled from two experiments. c CCR7 expression on CD4SP, Type A and Type B IELp from B6 and BCL2-tg mice, representative of two experiments.

To test whether TCR $\beta$ transgenes affect apoptosis efficiency during wave 1 deletion, we compared the geometric means of the TCR $\beta+$ CD5 + Helios + CCR7cell frequencies among EdU $+\mathrm{CD} 24+$ thymocytes in the presence or absence of BCL2-tg expression (Fig. 3f). In B6 mice the data were consistent with apoptosis of $92 \%$ of would-be Helios + thymocytes, calculated by subtracting the apoptosis-sufficient group mean of $0.23 \%$ from the BCL2-tg group mean of 3.0\%. Similar estimates were obtained for B3K506 -tg and Yae62 $\beta$-tg mice $(90 \%$ and $94 \%$, respectively), indicating that changes in cellular flow through the wave 1 checkpoint can occur with little change in apoptosis efficiency. d Percentage of unique TCR $\alpha$ or TCR $\beta$ sequences (denoted above graphs) with a self-reactive CDR3 P6-P7 doublet (hydrophobic index) in T-cell subsets (see colour key, right) from BCL2-tg mice ( $y$-axis) versus B6 mice ( $x$-axis). e Percentage of unique sequences with cysteine within two positions of the CDR3 apex (cysteine index) presented as in (d). In (d) and (e), symbols show the group mean and error bars the SEM of $\geq 3$ mice per group. For each T-cell subset examined in (a), (b), (d) and (e), a Student's $t$ test was used to compare B6 and BCL2-tg groups followed by Holm-Sidak's post-test without assuming a consistent standard deviation. $P$ values: $*<0.05$; $* * * *<0.0001$. In (d) and (e), $P$ and $R^{2}$ values were determined using Pearson's test for correlation

\section{Usage of proximal, but diverse, TCRa gene segments in $\mathrm{T}$ cells expressing Yae62 $\beta$-tg}

To test whether the increased induction of TCR-signalled thymocytes in Yae62 $\beta$-tg mice depends on repetitive usage of particular TCR $\alpha$ variable (Trav) and TCR $\alpha$ joining (Traj) gene segments, we compared the diversity of Trav-Traj combinations expressed by thymocyte subsets. Rarefaction and extrapolation curves based on the observed number of TCR $\alpha$ clones (Fig. 4a) were converted to curves based on sample coverage (Fig. 4b) to enable the comparison of Trav-Traj diversity at an equal level of completeness [23, 24]. When apoptosis was normal, Trav-Traj diversity 


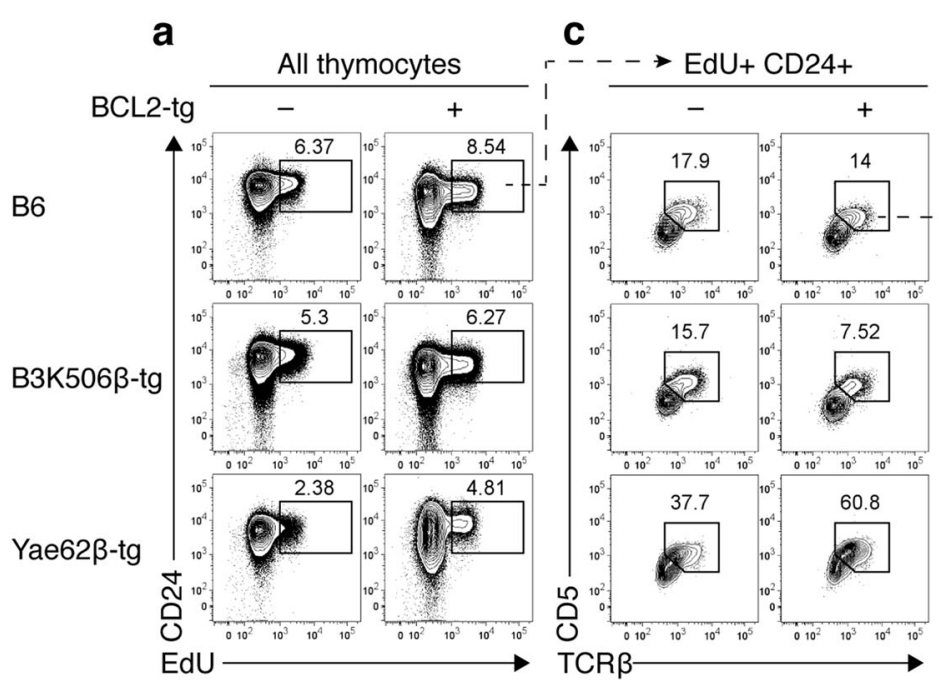

e
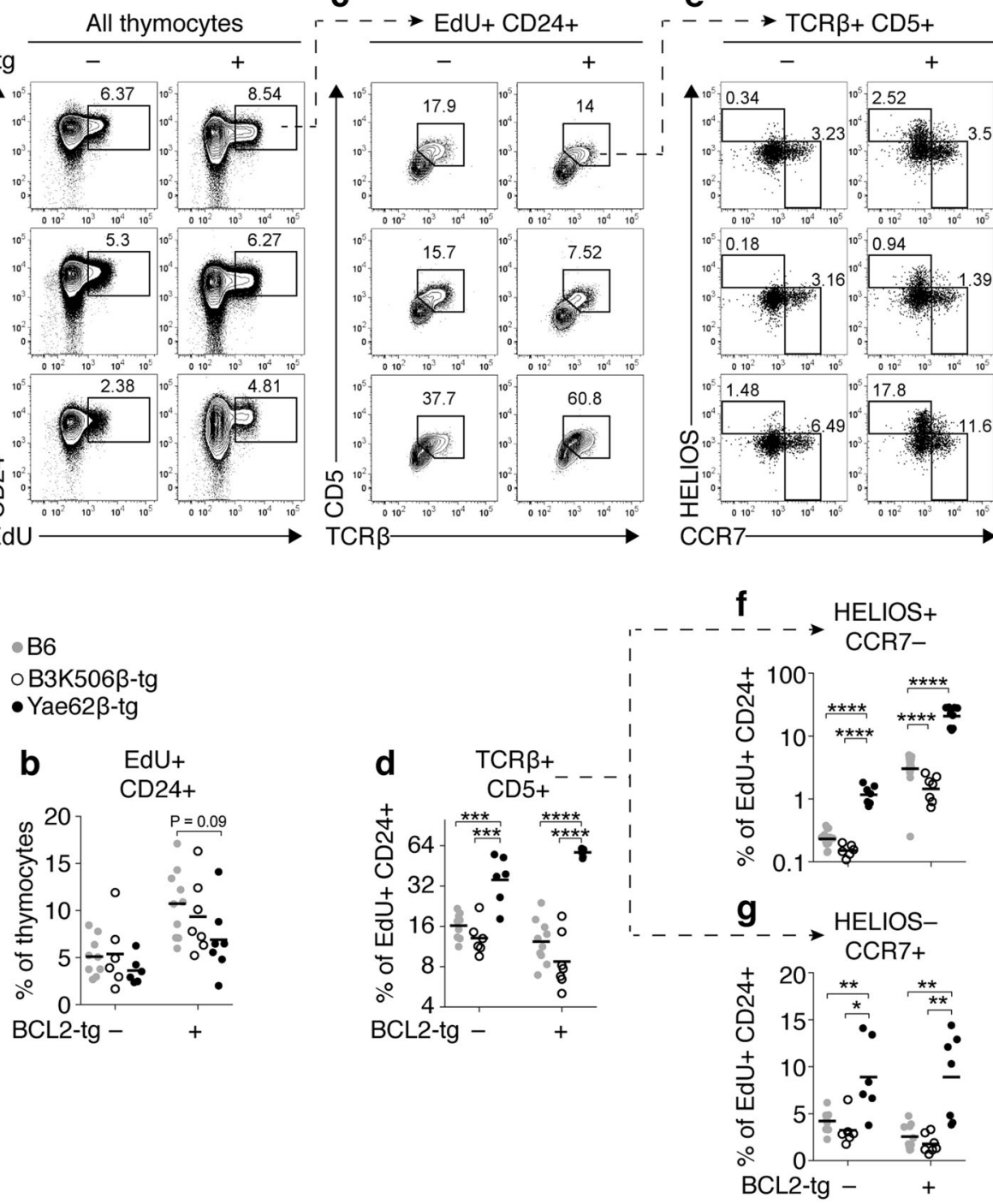

Fig. 3 Altered scales of wave 1 deletion in TCR $\beta$-transgenic mice. B6, B3K506 $\beta$-tg and Yae62 $\beta$-tg mice (denoted left), some of which carried BCL2-tg (denoted above plots), were injected once with EdU i.v. 3 days before thymocytes were analysed by flow cytometry. a Thymocytes gated to identify the EdU $+\mathrm{CD} 24+$ population, quantified for multiple mice in (b). $\mathbf{c}$ EdU $+\mathrm{CD} 24+$ thymocytes with a gate for the TCR $\beta+\operatorname{CD} 5+$ subset, quantified for multiple mice in $(\mathbf{d})$. e TCR $\beta+\mathrm{CD} 5+$ thymocytes gated to identify Helios + CCR7- and Helios-CCR7 + subsets, quantified in (f) and (g), respectively.

was highest in B6 mice, intermediate in B3K506 $\beta$-tg mice and lowest in Yae62 $\beta$-tg mice (Fig. 4c). Trav-Traj diversity in mice expressing both Yae62 $\beta$-tg and BCL2-tg exceeded that observed in Yae62 $\beta$-tg mice and was only slightly lower than that observed in mice expressing BCL2-tg alone (Fig. 4c). This finding suggests that the mechanism by which Yae62 $\beta$-tg increases the induction of TCR-signalled thymocytes does not depend on repetitive Trav-Traj combination usage.

As TCR $\alpha$ recombination events in DP thymocytes incorporate more distal Trav and Traj segments over time
Numbers on flow cytometry plots indicate the percentage of gated cells in the parent population, except for (e), which shows the percentage among the "grandparent" EdU + CD24 + population. Each symbol on one graph represents an individual mouse and lines show the group mean in (b) and (g) or geometric mean in (d) and (f). Data were compiled from four experiments. Student's $t$ tests were performed with Holm-Sidak's post-test without assuming a consistent standard deviation. Tests were performed on log-transformed values in (d) and (f). $P$ values: $*<0.05 ; * *<0.01 ; * * *<0.001 ; * * * * 0.0001$

[25], we examined the chromosomal distribution of TravTraj combinations. The Trav and Traj loci were each divided into five regions containing sets of gene segments used equally in B6 pre-selection thymocytes. Trav-Traj combinations were widely distributed along the Trav and Traj loci, including in Yae62 $\beta$-tg mice (Fig. 4d). However, close examination revealed proximal shifts in Yae62 $\beta$-tg mice compared with B6 mice in all thymocyte subsets (Supplementary Figure 3). This indicates that TCR $\alpha$ recombination time is relatively short in Yae62 $\beta$-tg mice, consistent with the increased induction of TCR-signalled thymocytes. 
a
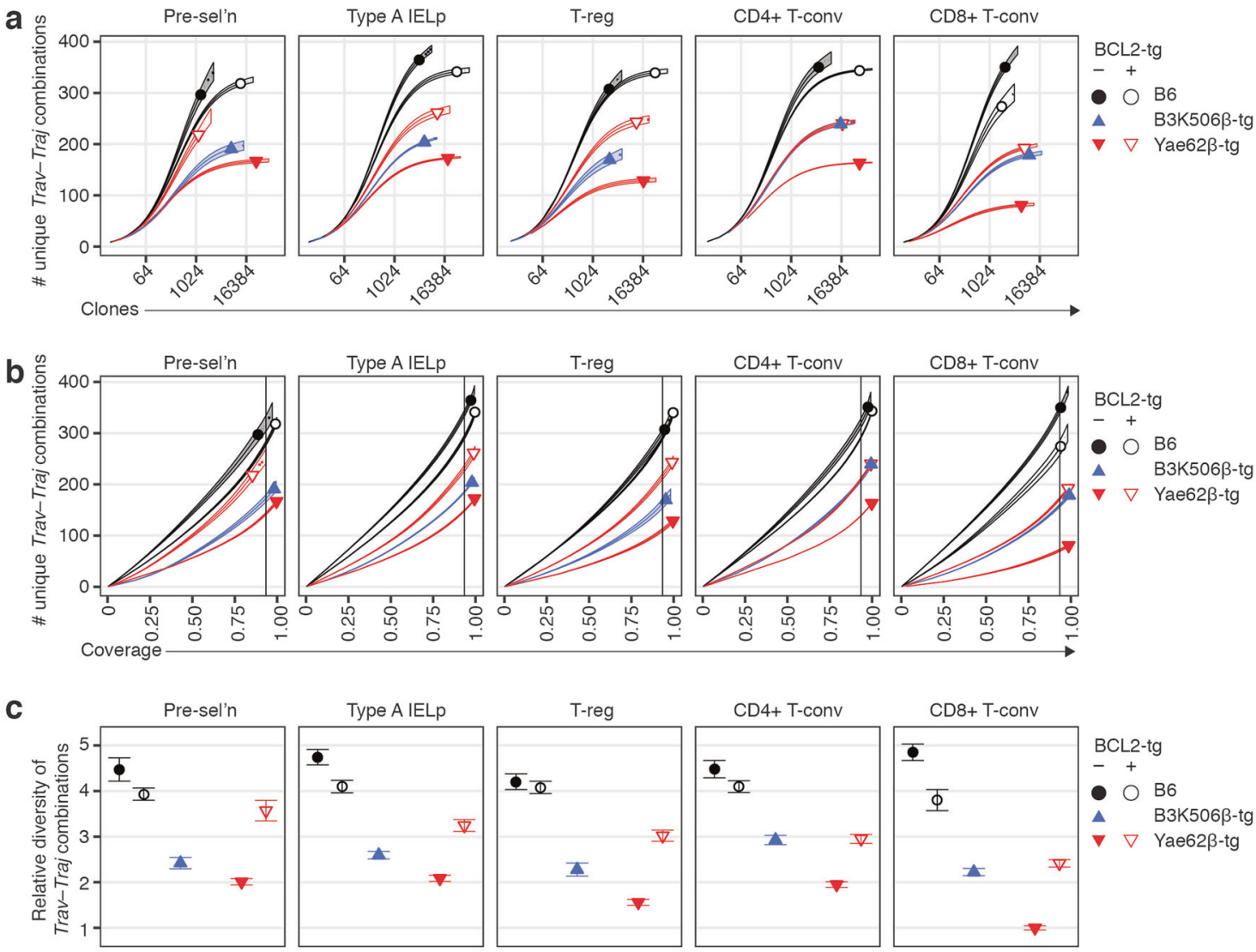

d

B6
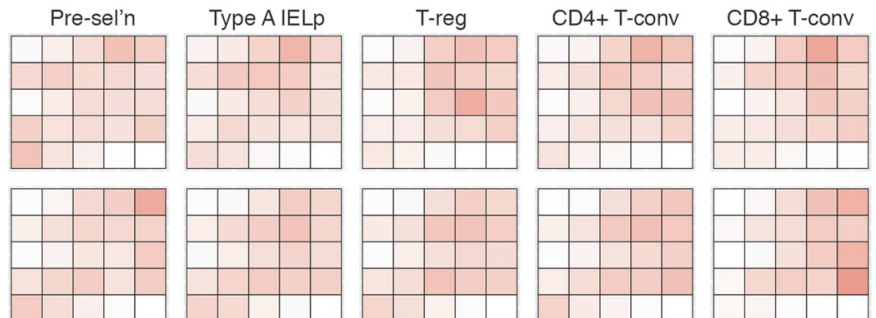

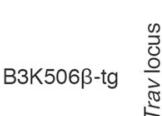
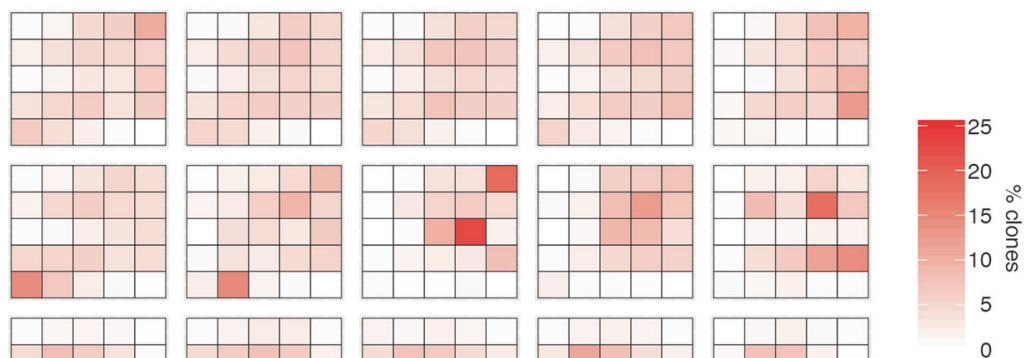

Yae62ß-tg
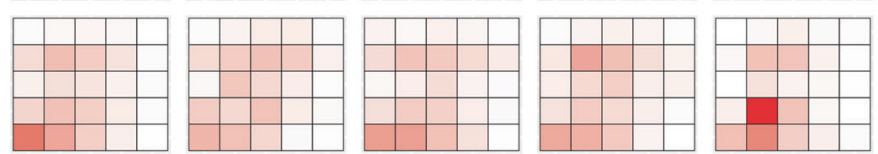

Yae62 $\beta$-tg

BCL2-tg
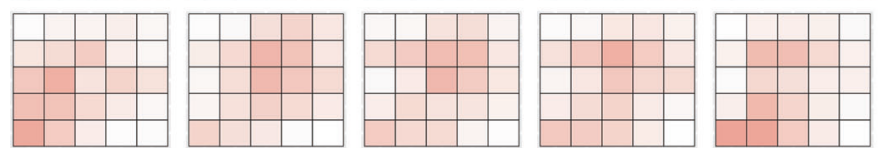

Trajlocus

Fig. 4 Diverse TCR $\alpha$ gene segment usage in Yae62 $\beta$-tg mice. a Rarefaction/extrapolation curves based on sample size. For each thymic T-cell subset (top), curves show the number of unique Trav-Traj combinations versus the number of TCR $\alpha$ clones. Shapes (right) show the observed values and shaded areas show 95\% confidence bands determined using five bootstrap replications. b Rarefaction/extrapolation curves based on sample coverage, defined as the proportion of all TCR $\alpha$ clones predicted to exist in a T-cell subset that use a Trav-Traj combination present in the TCR catalogue. As Trav-Traj combination diversity was calculated using the diversity order (Hill number or $q$ ) of 1 , Shannon entropy is equal to the

natural log of the $y$-axis value in (a) and (b) [24]. c Relative Trav-Traj combination diversities calculated at the level of coverage indicated by the vertical lines in (b) with error bars showing 95\% confidence bands. d Chromosomal distribution of Trav-Traj combinations. The Trav and Traj loci were each divided into five regions, resulting in a 25 -square grid for the classification of all possible Trav-Traj combinations. For each combination of mouse strain (left) and T-cell subset (top), heatmaps show the percentage (mean of $n \geq 3$ mice per group) of TCR $\alpha$ clones that used a Trav-Traj combination in each square of the 25-square grid 


\section{Wave 1 deletion in the absence of epithelial MHC expression}

Although the APC requirements for conventional T-cell differentiation are established, the APC types that mediate the extensive wave 1 deletion quantified above remain undefined. To investigate this, chimeric mice were generated in which haemopoietic (donor) cells and/or nonhaemopoietic (host) cells had a $B 2 \mathrm{~m}^{-1-} \mathrm{H} 2-\mathrm{Aa}^{-1-}$ genotype and therefore lacked $\mathrm{MHC}$ expression. Half of the mice received BCL2-tg donor cells. As one group of chimeras became unwell and were euthanized $\sim 4$ weeks after irradiation, this group was not examined in detail. As the EdU $+\mathrm{CD} 24+$ cell frequency did not differ significantly across the seven experimental groups (Fig. 5a, b), we determined the frequencies of TCR-signalled cell subsets among EdU $+\mathrm{CD} 24+$ thymocytes. TCR $\beta+\mathrm{CD} 5+$ cells were rare in chimeras lacking MHC expression completely, as expected (Fig. 5c, d).

On the BCL2-tg background TCR $\beta+\mathrm{CD} 5+$ cell induction was significantly lower in chimeras with $\mathrm{MHC}$ expression confined to host cells compared with chimeras with normal MHC expression (Fig. 5c, d). As the induction of both Helios-CCR7 + and Helios + CCR7- cells was decreased by $40-45 \%$ (Fig. 5e-g), haemopoietic APC are crucial for normal cellular flow through the wave 1 checkpoint.

$\mathrm{TCR} \beta+\mathrm{CD} 5+$ cell induction tended to be lower in chimeras with MHC expression confined to donor cells compared with chimeras with normal MHC expression, without reaching statistical significance (Fig. 5c, d). However, Helios-CCR7 + cell induction was reduced by 20fold on the apoptosis-sufficient background and by fivefold on the BCL2-tg background (Fig. 5e, g). This was expected because naive $\mathrm{T}$-cell development requires thymocytes to interact with self-antigen/MHC ligands expressed by cTEC $[4,5]$. Surprisingly, Helios + CCR7- thymocyte induction was undiminished in chimeras with MHC expression confined to donor cells (Fig. 5e, f). This was unexpected because thymocytes were thought to require TCR engagement with self-antigen/MHC ligands expressed by cTEC to pass the death-by-neglect checkpoint [4-6].

\section{Normal Type A IELp population size in the absence of epithelial MHC expression}

Next, we investigated the extent of thymocyte differentiation in the chimeric mice described above by enumerating mature post-selection thymocyte subsets (Fig. 6a). The thymic T-reg population was smaller in all groups lacking MHC expression in host and/or donor cells (Fig. 6b), consistent with crucial contributions of multiple APC subsets to thymic T-reg selection [26]. The CD8SP population was also decreased in all groups lacking MHC expression in host and/or donor cells (Fig. 6c), whereas the CD4SP population was smaller only in groups lacking MHC expression in host cells (Fig. 6d). The Type A IELp population differed from other post-selection subsets, only showing a significant decrease in the group lacking MHC expression completely (Fig. 6e).

\section{Discussion}

An estimated $3 \%$ of thymocytes that receive strong TCR signalling at the CCR7-/DP stage reach peripheral lymphoid tissues [17]. Most of this cell loss is attributable to wave 1 deletion, which we estimate induces apoptosis in $\sim 90 \%$ of CCR7- thymocytes stimulated to upregulate Helios. Despite increased induction of TCR-signalled thymocytes in Yae62 $\beta$-tg mice, corroborated by proximal shifts in Trav and Traj usage, apoptosis efficiency during wave 1 deletion was normal in Yae62 $\beta$-tg mice. The scale of wave 1 deletion was small in the absence of MHC expression, but was surprisingly large when MHC expression was confined to either haemopoietic or non-haemopoietic cells. We conclude that an efficient, and at least partially redundant, APC network mediates wave 1 deletion.

As the enlarged Type A IELp population in BCL2-tg mice contains Helios + CCR7- cells rescued from apoptosis, the TCR repertoire of these cells provides an opportunity to sample the TCR repertoire eliminated by wave 1 deletion. TCR self-reactivity indices were significantly higher in Type A IELp than in T-reg, CD4 + T-conv and CD8 + T-conv, consistent with the flow-cytometric, timecourse evidence that Type A IELp differentiation and wave 1 deletion are the first mechanisms that induce tolerance in strongly self-reactive $\alpha \beta \mathrm{T}$ cells. Currently, the only clear distinction between Type A IELp differentiation and wave 1 deletion in the thymic cortex is whether the self-reactive thymocyte survives or dies [20,27]. As younger DP thymocytes survived TCR engagement better than older DP thymocytes in vitro, it was proposed that Type A IELp differentiation potential is lost as DP thymocytes age [28]. Preferential rescue of older DP thymocytes from wave 1 deletion should cause a distal shift in Trav and Traj usage in Type A IELp. Contrary to this prediction, we found a proximal shift in Trav usage, and no shift in Traj usage, in Type A IELp from BCL2-tg mice compared with B6 mice (Supplementary Figure 3). The TCR repertoire eliminated by wave 1 deletion appears remarkably similar to the TCR repertoire that induces Type A IELp differentiation.

Unlike Type A IELp, the TCR signal strength required for Type B IELp differentiation is unclear. In Nur77 $7^{\text {GFP }}$ mice, in which GFP expression is proportional to TCR signal strength [29], Type B IELp have low GFP expression 


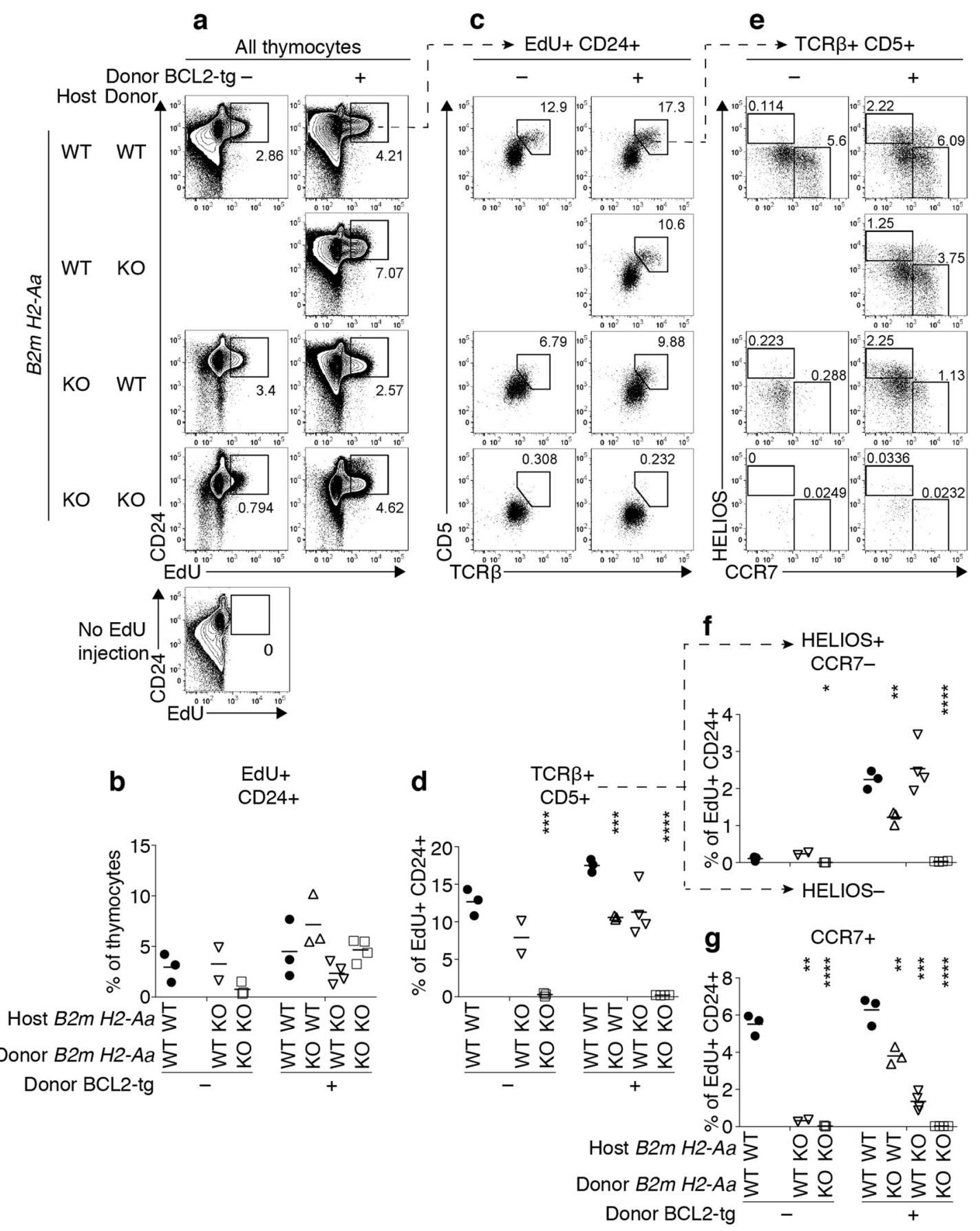

Fig. 5 Wave 1 deletion in the absence of epithelial MHC expression. Chimeras were generated by reconstituting irradiated B6 (WT) or $B 2 \mathrm{~m}^{-1-} \mathrm{H} 2-\mathrm{Aa}^{-/-}(\mathrm{KO})$ mice with WT or KO bone marrow cells (left). In addition, some mice received bone marrow cells that carried the BCL2 transgene (top). Chimeras were injected once with EdU i.p. 3 days before thymocytes were analysed at a time point 5 weeks after irradiation. No EdU + events were detected in thymocyte samples from three mice, presumably due to failure of the intraperitoneal injection; these samples were excluded. a Thymocytes gated to identify the EdU $+\mathrm{CD} 24+$ population, quantified for multiple chimeras in (b). $\mathbf{c} \mathrm{EdU}+\mathrm{CD} 24+$ thymocytes with a gate for the TCR $\beta+\mathrm{CD} 5+$ subset, quantified for multiple chimeras in (d). e TCR $\beta+$ CD $5+$ thymocytes gated to identify Helios + CCR7- and Helios-CCR7 + subsets, quantified in (f) and (g), respectively. Numbers on flow cytometry plots indicate the frequency of each subset among cells in the parent gate, except for (e), in which numbers show frequencies among the "grandparent" EdU + CD24 + population. Each symbol on one graph represents an individual mouse analysed in a single experiment. Student's $t$ tests were used to compare each group with the MHC-sufficient control group on the same background (apoptosissufficient or BCL2-tg) followed by Holm-Sidak's post-test without assuming a consistent standard deviation. $P$ values: $*<0.05$; $* *<0.01 ; * * *<0.001 ; * * * *<0.0001$ 
Fig. 6 Enumeration of postselection thymocyte subsets in chimeric mice lacking MHC expression in haemopoietic and/ or non-haemopoietic cells. a Thymocyte gating strategy applied to samples from chimeras described in Fig. 5, with summaries showing the number of cells per thymus in the following T-cell subsets: b T-reg, c CD8SP, d CD4SP and e Type A IELp. Each symbol on one graph represents an individual mouse analysed in a single experiment. Student's $t$ tests were used to compare logtransformed values for each group with the MHC-replete group on the same background (apoptosis-sufficient or BCL2$\mathrm{tg})$ followed by Holm-Sidak's post-test without assuming a consistent standard deviation. $P$ values: $* *<0.01$; $* * *<0.001 ; * * * *<0.0001$ a

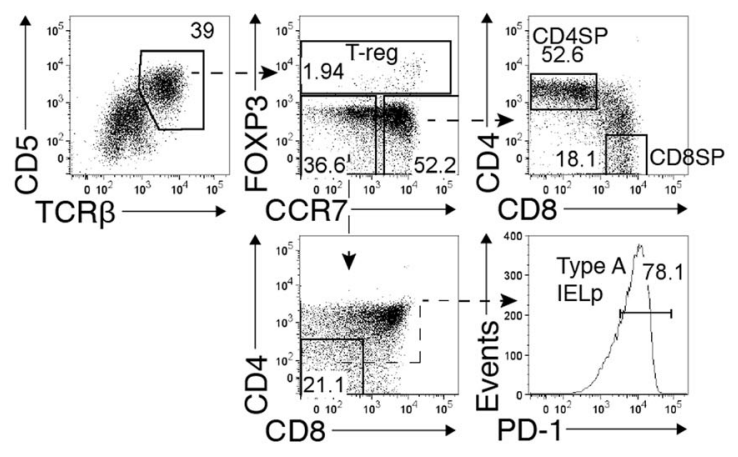

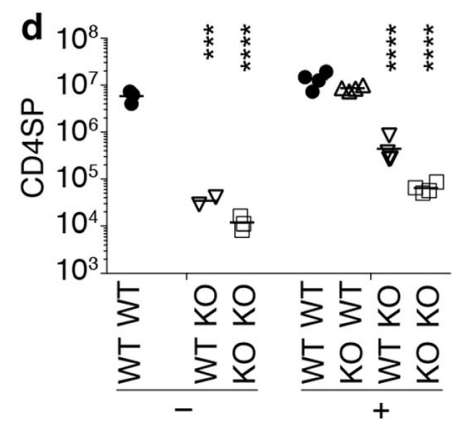
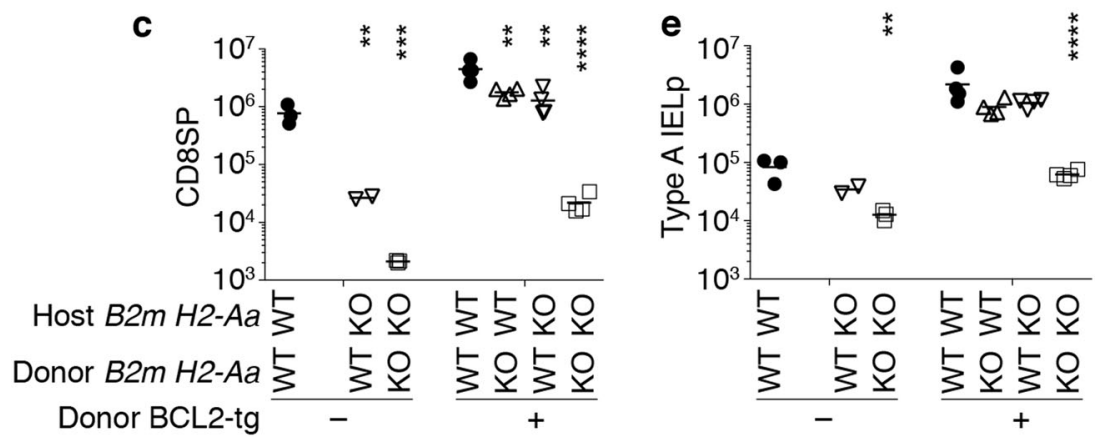

similar to non-TCR-signalled DP thymocytes $[19,20]$. Nur77 is also not expressed in the small subset of DN TCR $\beta+$ T-bet + thymocytes that do not express NK1.1 [30], the likely precursors of Type B IELp. However, these cells have high Helios expression, indicative of strong TCR signalling [30]. This uncoupling of Nur77 and Helios expression, two markers of TCR activation, in cells initiating Type B IELp differentiation remains unexplained. BCL2-tg expression increased the frequency of Helios + CCR7- cells and TCR self-reactivity indices in the Type B IELp population, suggesting that cells rescued from wave 1 deletion can differentiate into Type B IELp. Unlike our findings in Type A IELp, the data suggest that wave 1 deletion preferentially eliminates would-be Type B IELp expressing strongly self-reactive TCRs.

The CD4 + T-conv TCR $\beta$ repertoire selected in the presence of BCL2-tg expression had a higher hydrophobic index than controls, as observed in mice with defective apoptosis due to the absence of Bim [22]. The cysteine indices in the $\mathrm{CD} 4+\mathrm{T}$-conv $\mathrm{TCR} \alpha$ and $\mathrm{TCR} \beta$ repertoires were also increased in BCL2-tg mice compared with B6 mice. As an increased cysteine index is a distinctive feature of IELp and IEL, some thymocytes that would normally acquire tolerance in the thymic cortex would seem to escape that process in BCL2-tg mice. The excessive number of undeleted self-reactive thymocytes in the cortex of mice with defective apoptosis [7,31] may increase competition for thymic APC to the point that some self-reactive thymocytes escape cortical tolerance mechanisms. The CD4 + T-conv TCR repertoire seems to be a sensitive domain for detecting increased TCR self-reactivity because the lowest TCR self-reactivity indices in normal post-selection T-cell subsets are found in CD4 + T-conv. This may seem counterintuitive given that Nur $77^{\mathrm{GFP}}$ expression is higher in CD4 + T-conv than in CD8 + T-conv [29], but can be explained by the finding that thymocytes are more sensitive to MHCII compared with MHCI because more CD4 coreceptors are Lck-loaded compared with CD8 [32]. 
When MHC expression was confined to nonhaemopoietic cells, the T-reg and CD8SP populations were diminished. Haemopoietic APC are essential for normal T-reg development [26], but a non-redundant role for haemopoietic APC in CD8SP development was unexpected and requires further attention. On the other hand, given the crucial role of haemopoietic APC in deletion [5, 33], it was surprising that wave 1 deletion was only halved when MHC expression was confined to non-haemopoietic cells. This might indicate that non-haemopoietic APC can induce wave 1 deletion in self-reactive thymocytes, albeit on a smaller scale than in the normal thymus. Non-haemopoietic APC appear sufficient to induce thymocytes to attain wave 1 deletion phenotypes, such as low CD4/CD8 expression and high expression of PD-1 [27] and Helios [34]. However, intercellular self-antigen/MHC transfer from nonhaemopoietic cells to haemopoietic cells [35] may also underlie wave 1 deletion in these settings.

We found that many thymocytes passed the death-byneglect checkpoint in chimeric mice with MHC expression confined to haemopoietic cells. The ability of haemopoietic cells to autonomously induce wave 1 deletion was overlooked [5] because those experiments predate methods of distinguishing wave 1 deletion from death by neglect. After such methods were developed, it was still assumed that passing the death-by-neglect checkpoint requires TCR engagement with self-peptide/MHC ligands on nonhaemopoietic cells [6]. The data do not exclude an essential role for non-haemopoietic cells, for example acting as a scaffold on which thymocytes can engage other haemopoietic cells, but our data show that MHC expression in haemopoietic cells is sufficient for a normal amount of wave 1 deletion. By contrast, the induction of TCR-signalled Helios-CCR7 + thymocytes was markedly decreased when MHC expression was confined to haemopoietic cells, consistent with the essential role of thymic epithelial cells as inducers of naive T-cell differentiation [4, 5]. Altogether, cell type-specific ablation of $\mathrm{MHC}$ expression impairs conventional T-cell differentiation to a greater extent than it impairs wave 1 deletion in the thymus.

\section{Materials and methods}

\section{Mice}

C57BL/6 (B6), Tg(Vav-BCL2) ${ }^{1 \mathrm{Jad}}$ (BCL2-Tg), H2-Aa ${ }^{\mathrm{tm} 1 \mathrm{Blt}}$, $B 2 m^{\text {tm1Jae }}$ Yae62 $\beta$-tg, B3K506 $\beta$-tg, Foxp3 ${ }^{\text {tml.1Ayr }}$ $\left(\right.$ Foxp $\left.3^{G F P}\right)$ mice, all on the B6 genetic background, were bred (intercrossed in some cases) and housed at the Australian Phenomics Facility, Canberra or at Monash University, Melbourne, and were genotyped using PCR assays on genomic DNA extracted from ear or tail biopsies. Some
Yae62 $\beta$-tg and B3K506 $\beta-\operatorname{tg}$ mice were heterozygous for the Tcra $^{\text {null }}$ (Tcra ${ }^{\text {Tm1Mom }}$ ) allele. T-cell subsets for TCRsequencing were sorted from Foxp $3^{G F P}$ mice. To make chimeras, recipient mice were irradiated with X-rays (two doses of $4.5 \mathrm{~Gy}$ given $4 \mathrm{~h}$ apart) and then injected intravenously with at least $2 \times 10^{6}$ bone marrow cells. The Animal Experimentation Ethics Committees of the Australian National University or Monash University approved all procedures.

\section{EdU labelling and flow cytometry}

In total, $0.25 \mathrm{mg}$ EdU in PBS was injected i.v. or i.p. at varying times before analysis as described in figure legends. For CCR7 staining, single cell thymocyte suspensions were incubated for $60 \mathrm{~min}$ at $37^{\circ} \mathrm{C}$ in pre-warmed FACS buffer (PBS containing 2\% v/v heat-inactivated bovine serum and $0.01 \% \mathrm{~m} / \mathrm{v}$ sodium azide) containing fluorochrome- or biotin-conjugated anti-CCR7 (BioLegend, San Diego, CA, Cat \#120104 or 120105). Cells were then pelleted by centrifugation and incubated for $30 \mathrm{~min}$ in FACS buffer at $4{ }^{\circ} \mathrm{C}$ containing assortments of antibodies against TCR $\beta$ (BioLegend, Cat \#109233 or \#109234), CD5 (Miltenyi, Bergisch Gladbach, Germany, Order \#130-103-796), CD4 (BioLegend, Cat \#100430), CD8 $\alpha$ (BioLegend, Cat \#100766), PD-1 (BioLegend, Cat \#135218), CD25 (BioLegend, Cat \#102004) or NK1.1 (BD Pharmingen, San Jose, CA, Cat \#553164) and PE- or BV421-conjugated tetramers of mouse CD1d loaded with PBS-44 glycolipid. After washing in FACS buffer, cells were fixed and permeabilised using the Foxp3/ ranscription Factor Staining Buffer Set (Thermo Fisher Scientific, Waltham, MA, Cat \#00-5523-00), then incubated with antibodies specific for Helios (BioLegend, Cat \#137220 or \#137222) and Foxp3 (Thermo Fisher, Cat \#11-5773-80). Samples were then processed using the Click-iT ${ }^{\circledR}$ EdU Flow Cytometry Assay Kit (Thermo Fisher Scientific, Cat \#C10420 or \#C10635) following the manufacturer's instructions except that Click$\mathrm{iT}^{\circledR}$ EdU buffer additive (Component $\mathrm{G}$ ) was used at onefifth of the concentration recommended. Samples were then washed in FACS buffer and incubated with anti-CD24 (Miltenyi, Order \#130-102-736) and streptavidin-PE or -BV650 (BioLegend, Cat\# 405204 or \#405232). After washing in FACS buffer, data were acquired with LSR II flow cytometers (Becton Dickinson, Franklin Lakes, New Jersey) and analysed using FlowJo software (FlowJo LLC, Ashland, Oregon).

\section{T-cell sorting}

Methods used to sort T-cell subsets were described previously [21]. To sort Type B IELp, thymocytes were additionally stained with propidium iodide or live/dead fixable aqua (Thermo Fisher, Cat \#L34965), tetramers of mouse CD1d loaded with PBS-44 glycolipid and antibodies 
against CD5, TCR $\beta, \gamma \delta$ TCR (BioLegend, Cat \#118103 or \#118131) and NK1.1 (Miltenyi, Order \#130-102-991).

\section{TCR-sequence acquisition, filtering and availability}

Methods used for RNA isolation, cDNA synthesis, PCR amplification of TCR $\alpha$ and TCR $\beta$ transcripts, addition of sequencing adapters and sample indices, amplicon concentration, purification, sequencing and alignment to mouse genome using molecular identifier groups-based error correction (MIGEC) software [36] were described previously [21], except that the set of Trav-specific forward primers was not identical in all experiments as detailed in Supplementary Table 1 . Sequences with a CDR3 that was out-offrame or contained a stop codon were excluded. To avoid overestimating TCR diversity due to PCR or sequencing errors, sequences detected only once in any given sample were excluded. A clonotype was defined as a unique combination of $\mathrm{V}$ gene and CDR3 amino acid sequence. Each clonotype was counted only once per sample. Due to duplication events at the mouse TCR $\alpha \delta$ locus, some reads aligned to $>1$ Trav paralog. Sequences that mapped to $>1$ Trav paralog, or were amplified from primers that were not used in every experiment, were excluded from Trav-Traj analyses but were included in analyses that required only CDR3 sequence information (hydrophobic and cysteine indices). The filtered dataset, with a column indicating whether each sequence was included in Trav-Traj analyses, is available at Monash University data repository using the Digital Object Identifier: 10.26180/5c6400f45472e.

\section{TCR-sequence analyses}

Trav-Traj diversity calculations were performed at the level of TCR catalogue, defined as the aggregate of all clonotypes detected in a given combination of mouse strain and T-cell subset. Clonotype abundance in a TCR catalogue equals the number of mice in which the clonotype was detected. The number of clones in a TCR catalogue is the sum of the abundances of all clonotypes. The iNEXT software package was used to produce sample size-based and coverage-based rarefaction and extrapolation curves using the diversity order (Hill number or $q$ ) of 1, with $95 \%$ confidence bands based on five bootstrap replications [24]. Relative diversity estimates were performed following the approach of Chao and Jost [23], whose term "individual" we equate with "clone", and "species" we equate with "Trav-Traj combination". Trav and Traj gene chromosomal locations were taken from ImMunoGeneTics (http://www.imgt.org, Locus gene order) [37]. For hydrophobic and cysteine index analyses, CDR3 sequences $<8$ amino acids were excluded because a conserved Phe or Try is present at position 6 or 7 of CDR3 sequences that are 6 or 7 amino acids long. The hydrophobic index equals the percentage of unique clonotypes with a CDR3 P6-P7 doublet corresponding to any of the 175 amino acid doublets identified as promoting selfreactivity when present at CDR3 $\beta$ P6-P7 [22]. The cysteine index equals the percentage of unique clonotypes with cysteine within two positions of the CDR3 apex. Briefly, for a CDR3 sequence of $n$ amino acids, the amino acid at the largest position not greater than $(n / 2+1)$ was defined as the CDR3 apex [21]. As the TCR $\alpha$ repertoires of two Type B IELp samples from B6 mice had zero clonotypes with cysteine within two positions of the CDR3 apex, the cysteine index of these samples was defined as the reciprocal of the number of clonotypes in the sample, expressed as a percentage.

\section{Statistical analyses}

The "tidyverse", "stringr" and "reshape2" packages were used in RStudio software for TCR-sequence analyss, to produce graphs and to perform Pearson's tests for correlation. Other statistical analyses were performed using GraphPad Prism version 7.0a (GraphPad Software, La Jolla, California, USA) with multiple comparisons tests recommended in the GraphPad Prism software. Figures were made using Adobe Illustrator CC (Adobe Systems Inc., San Jose, CA).

Acknowledgements We thank Eric Huseby (University of Massachusetts Medical School) for the gift of the Yae62 $\beta$-tg and B3K506 $\beta$ tg mice, Dale Godfrey and Marcin Ciula for the PBS44/CD1d tetramers and the staff of Monash Animal Research Platform, FlowCore and Micromon for technical assistance. This research was supported by the Monash Biomedicine Discovery Institute, by the National Health and Medical Research Council Grant 1107464 to SRD, Grant 1016953 and Australia Fellowship 585490 to CCG.

\section{Compliance with ethical standards}

Conflict of interest The authors declare that they have no conflict of interest.

Publisher's note: Springer Nature remains neutral with regard to jurisdictional claims in published maps and institutional affiliations.

\section{References}

1. Daley SR, Teh C, Hu DY, Strasser A, Gray DHD. Cell death and thymic tolerance. Immunol Rev. 2017;277:9-20.

2. Daley SR, Hu DY, Goodnow CC. Helios marks strongly autoreactive $\mathrm{CD} 4+\mathrm{T}$ cells in two major waves of thymic deletion distinguished by induction of PD-1 or NF- $\kappa$ B. J Exp Med. 2013;210:269-85.

3. Hu DY, Yap JY, Wirasinha RC, Howard DR, Goodnow CC, Daley SR. A timeline demarcating two waves of clonal deletion and Foxp3 upregulation during thymocyte development. Immunol Cell Biol. 2016;94:357-66.

4. Cosgrove D, Chan SH, Waltzinger C, Benoist C, Mathis D. The thymic compartment responsible for positive selection of CD4 + T cells. Int Immunol. 1992;4:707-10. 
5. van Meerwijk JP, Marguerat S, Lees RK, Germain RN, Fowlkes BJ, MacDonald HR. Quantitative impact of thymic clonal deletion on the T cell repertoire. J Exp Med. 1997;185:377-83.

6. Sinclair C, Bains I, Yates AJ, Seddon B. Asymmetric thymocyte death underlies the CD4:CD8 T-cell ratio in the adaptive immune system. Proc Natl Acad Sci USA. 2013;110:E2905-14.

7. Stritesky GL, Xing Y, Erickson JR, Kalekar LA, Wang X, Mueller DL, et al. Murine thymic selection quantified using a unique method to capture deleted T cells. Proc Natl Acad Sci USA. 2013;110:4679-84.

8. Strasser A, Harris AW, von Boehmer H, Cory S. Positive and negative selection of $\mathrm{T}$ cells in T-cell receptor transgenic mice expressing a bcl-2 transgene. Proc Natl Acad Sci USA. 1994;91: 1376-80.

9. Stadinski BD, Trenh P, Smith RL, Bautista B, Huseby PG, Li G, et al. A role for differential variable gene pairing in creating $\mathrm{T}$ cell receptors specific for unique major histocompatibility ligands. Immunity. 2011;35:694-704.

10. Penit C, Lucas B, Vasseur F. Cell expansion and growth arrest phases during the transition from precursor (CD4-8-) to immature $(\mathrm{CD} 4+8+)$ thymocytes in normal and genetically modified mice. J Immunol. 1995;154:5103-13.

11. Penit C. Localization and phenotype of cycling and post-cycling murine thymocytes studied by simultaneous detection of bromodeoxyuridine and surface antigens. J Histochem Cytochem. 1988;36:473-8.

12. Lucas B, Vasseur F, Penit C. Normal sequence of phenotypic transitions in one cohort of 5-bromo-2'-deoxyuridine-pulselabeled thymocytes. Correlation with $\mathrm{T}$ cell receptor expression. $\mathrm{J}$ Immunol. 1993;151:4574-82.

13. Ogilvy S, Metcalf D, Print CG, Bath ML, Harris AW, Adams JM. Constitutive $\mathrm{Bcl}-2$ expression throughout the hematopoietic compartment affects multiple lineages and enhances progenitor cell survival. Proc Natl Acad Sci USA. 1999;96:14943-8.

14. Saini M, Sinclair C, Marshall D, Tolaini M, Sakaguchi S, Seddon B. Regulation of Zap70 expression during thymocyte development enables temporal separation of $\mathrm{CD} 4$ and $\mathrm{CD} 8$ repertoire selection at different signaling thresholds. Sci Signal. 2010;3:ra23.

15. Mayans S, Stepniak D, Palida SF, Larange A, Dreux J, Arlian $\mathrm{BM}$, et al. $\mathrm{T}$ cell receptors expressed by $\mathrm{CD} 4^{-} \mathrm{CD} 8 \alpha \beta^{-}$intraepithelial $\mathrm{T}$ cells drive their fate into a unique lineage with unusual MHC reactivities. Immunity. 2014;41:207-18.

16. McDonald BD, Bunker JJ, Erickson SA, Oh-Hora M, Bendelac A. Crossreactive $\alpha \beta \mathrm{T}$ cell receptors are the predominant targets of thymocyte negative selection. Immunity. 2015;43:859-69.

17. McDonald BD, Bunker JJ, Ishizuka IE, Jabri B, Bendelac A. Elevated $\mathrm{T}$ cell receptor signaling identifies a thymic precursor to the $\mathrm{TCR} \alpha \beta^{+} \mathrm{CD} 4^{-} \mathrm{CD} 8 \beta^{-}$intraepithelial lymphocyte lineage. Immunity. 2014;41:219-29.

18. Pobezinsky LA, Angelov GS, Tai X, Jeurling S, Van Laethem F, Feigenbaum $\mathrm{L}$, et al. Clonal deletion and the fate of autoreactive thymocytes that survive negative selection. Nat Immunol. 2012;13:569-78.

19. Golec DP, Hoeppli RE, Henao Caviedes LM, McCann J, Levings MK, Baldwin TA. Thymic progenitors of TCR $\alpha \beta^{+}$CD8 $\alpha \alpha$ intestinal intraepithelial lymphocytes require RasGRP1 for development. J Exp Med. 2017;214:2421-35.

20. Ruscher R, Kummer RL, Lee YJ, Jameson SC, Hogquist KA. $\mathrm{CD} 8 \alpha \alpha$ intraepithelial lymphocytes arise from two main thymic precursors. Nat Immunol. 2017;18:771-9.
21. Wirasinha RC, Singh M, Archer SK, Chan A, Harrison PF, Goodnow CC, et al. T-cell receptors with a central CDR3 cysteine are enriched in CD8 $\alpha \alpha$ intraepithelial lymphocytes and their thymic precursors. Immunol Cell Biol. 2018;96:553-61.

22. Stadinski BD, Shekhar K, Gomez-Tourino I, Jung J, Sasaki K, Sewell AK, et al. Hydrophobic CDR3 residues promote the development of self-reactive T cells. Nat Immunol. 2016;17: 946-55.

23. Chao A, Jost L. Coverage-based rarefaction and extrapolation: standardizing samples by completeness rather than size. Ecology. 2012;93:2533-47.

24. Chao A, Gotelli NJ, Hsieh TC, Sander EL, Ma KH, Colwell RK, et al. Rarefaction and extrapolation with Hill numbers: a framework for sampling and estimation in species diversity studies. Ecol Monogr. 2014;84:45-67.

25. Guo J, Hawwari A, Li H, Sun Z, Mahanta SK, Littman DR, et al. Regulation of the TCR $\alpha$ repertoire by the survival window of $\mathrm{CD} 4{ }^{+} \mathrm{CD} 8^{+}$thymocytes. Nat Immunol. 2002;3:469-76.

26. Perry JS, Lio CW, Kau AL, Nutsch K, Yang Z, Gordon JI, et al. Distinct contributions of Aire and antigen-presenting-cell subsets to the generation of self-tolerance in the thymus. Immunity. 2014;41:414-26.

27. McCaughtry TM, Baldwin TA, Wilken MS, Hogquist KA. Clonal deletion of thymocytes can occur in the cortex with no involvement of the medulla. J Exp Med. 2008;205:2575-84.

28. Verstichel G, Vermijlen D, Martens L, Goetgeluk G, Brouwer M, Thiault N, et al. The checkpoint for agonist selection precedes conventional selection in human thymus. Sci Immunol. 2017;2: eaah4232.

29. Moran AE, Holzapfel KL, Xing Y, Cunningham NR, Maltzman JS, Punt J, et al. T cell receptor signal strength in Treg and iNKT cell development demonstrated by a novel fluorescent reporter mouse. J Exp Med. 2011;208:1279-89.

30. Klose CSN, Hummel JF, Faller L, d'Hargues Y, Ebert K, Tanriver Y. A committed postselection precursor to natural $\mathrm{TCR} \alpha \beta^{+}$intraepithelial lymphocytes. Mucosal Immunol. 2018;11: 333-44.

31. Kovalovsky D, Pezzano M, Ortiz BD, Sant'Angelo DB. A novel TCR transgenic model reveals that negative selection involves an immediate, Bim-dependent pathway and a delayed, Bimindependent pathway. PLoS ONE. 2010;5:e8675.

32. Stepanek O, Prabhakar AS, Osswald C, King CG, Bulek A, Naeher $\mathrm{D}$, et al. Coreceptor scanning by the $\mathrm{T}$ cell receptor provides a mechanism for T cell tolerance. Cell. 2014;159:333-45.

33. Huseby ES, Crawford F, White J, Kappler J, Marrack P. Negative selection imparts peptide specificity to the mature $\mathrm{T}$ cell repertoire. Proc Natl Acad Sci USA. 2003;100:11565-70.

34. Yap JY, Wirasinha RC, Chan A, Howard DR, Goodnow CC, Daley SR. Indirect presentation in the thymus limits naive and regulatory $\mathrm{T}$-cell differentiation by promoting deletion of selfreactive thymocytes. Immunology. 2018;154:522-32.

35. Koble C, Kyewski B. The thymic medulla: a unique microenvironment for intercellular self-antigen transfer. J Exp Med. 2009;206:1505-13.

36. Shugay M, Britanova OV, Merzlyak EM, Turchaninova MA, Mamedov IZ, Tuganbaev TR, et al. Towards error-free profiling of immune repertoires. Nat Methods. 2014;11:653-5.

37. Bosc N, Lefranc MP. The mouse (Mus musculus) T cell receptor alpha (TRA) and delta (TRD) variable genes. Dev Comp Immunol. 2003;27:465-97. 